\title{
Accuracy of Trace Formulas
}

\author{
Arul Lakshminarayan \\ Physical Research Laboratory, \\ Navarangapura, Ahmedabad, 380009, India.
}

\begin{abstract}
Using quantum maps we study the accuracy of semiclassical trace formulas. The role of chaos in improving the semiclassical accuracy, in some systems, is demonstrated quantitatively. However, our study of the standard map cautions that this may not be most general. While studying a sawtooth map we demonstrate the rather remarkable fact that at the level of the time one trace even in the presence of fixed points on singularities the trace formula may be exact, and in any case has no logarithmic divergences observed for the quantum bakers map. As a byproduct we introduce fantastic periodic curves akin to curlicues.
\end{abstract}

Keywords: Quantum Chaos, Quantum Maps, Gutzwiller's Periodic Orbit Sum.

Submitted to Pramana, Journal of Physics, (Special Issue on Nonlinearity and Chaos in Physical Sciences.) 


\section{Introduction}

The trace formula of Gutzwiller [1] provides a window into the semiclassical treatment of chaotic systems and is hence of considerable importance in many areas of physics and chemistry. Yet the understanding of this formula has been hampered by the complexity of the classical task involved, and often the semiclassical effort is greater than that for a full quantum solution which makes the semiclassical methods either only of academic interest or as tools for explaining features of a full quantum solution. In this situation simple models have played a key role just as has been the case with the understanding of classical chaos, as evidenced for example by the study of the logistic map and the smale horseshoe.

Although the trace formula (and its relatives) are important, the accuracy of these have not been rigorously established. The trace formula being the first term in an asymptotic expansion, has many approximations with apparently uncontrolled errors. The very many applications of the stationary phase approximation, on functional and on ordinary integrals obscures the nature of the errors. One ongoing issue is the time at which we can expect the various semiclassical approximations to go bad with a candidate the so called log time which is of the order of $-\log (\hbar) / \lambda$, applicable for chaotic systems with a Lyapunov exponent $\lambda$, being called too pessimistic [2]. We note that although the semiclassical formula for the time evolution (due to Van Vleck) has been essentially known almost since the beginning of quantum mechanics the nature of the errors is still a matter of interest, especially since the trace formula depends on a Laplace transform of the Van Vleck approximation [1].

The quantum bakers map has been studied as a model of quantum chaos as indeed it has many generic features of quantized chaotic systems $[3,4,5]$, however a rigorous study of some of the simplest traces of the quantum problem [5] showed logarithmic divergence from the expected Gutzwiller trace formula. The genesis of this disturbing fact was attributed to a fixed point on the line of discontinuity (corners) of the classical map. A baker without corners was studied [6], and it was found that there were no logarithmic divergences anymore. However we will in this note display a map, the sawtooth map, which has a fixed point on the line of discontinuity, but which has no logarithmic divergences. In fact we will show that in certain circumstances the semiclassical trace can be exactly the quantum trace.

In pursuance of certain results in [5], we use the existing models of quantum chaos to study the accuracy of the simplest trace of the quantum problem. To this end we will in particular use the much studied quantum standard map and the much less studied quantum sawtooth map both in the settings of the phase space as a cylinder and as a torus. These being maps derived from time periodic systems we study simply the trace of the relevant unitary Floquet operators. In section 2 the cylindrical phase space is used, while in section

3 the toral phase space is used, we end with some comments in section 4 . In the Appendix 
we provide a self contained derivation of the "trace formula" for a class of maps on the torus that includes the maps studied below and all the so called kicked systems. While this is in principle known, it may be useful to recollect it here, as the other published proof of Tabor [17] is for the case of maps on the entire plane, and is hence quite different. Those with little exposure to the trace formula may peruse the Appendix first.

\section{The Cylindrical Phase Space}

Consider the well known standard map $\left(q_{n+1}=q_{n}+T p_{n}, p_{n+1}=p_{n}+K \sin \left(q_{n+1}\right)\right)$ defined on the cylinder $[0,2 \pi) \times(-\infty, \infty)$ with periodic boundary conditions in $q$. The first iterative equation is them to be evaluated with a modulo $2 \pi$ rule. A simple change of variables from $p$ to $T p$ implies that the relevant parameter is just one in number and is $k=K T$. This area preserving map and its quantization have been studied in extensive detail as a model of chaos and its quantum manifestations and led to such important discoveries as the quantum suppression of chaotic diffusion. Some of these are reviewed in [7]. However its use in understanding the Gutzwiller trace formula has been limited. The work of Tabor [17] where he derived the relevant trace formula for quantum maps on the plane did use the standard map but no rigorous or extensive study has yet been attempted. The quantization of the standard map is described by an infinite dimensional unitary matrix with matrix elements in the momentum representation being

$$
U_{n m}=(-i)^{n-m} J_{n-m}(k / T \hbar) \exp \left(-i m^{2} \hbar T / 2\right) .
$$

Here $J_{\nu}(z)$ is a Bessel function. As has been noted earlier there are two relevant parameters quantum mechanically and we can take $T=1$ while we do not have the freedom to choose $\hbar=1$ anymore. The trace of the quantum map is then given by

$$
\operatorname{Tr}(U)=J_{0}(k / \hbar) \sum_{n=-\infty}^{\infty} e^{-i \hbar n^{2} / 2}=J_{0}(k / \hbar) \Theta_{3}(0 \mid-\hbar / 2 \pi)
$$

where $\Theta_{3}$ is a Jacobi theta function. An application of the Poisson formula enables us to rewrite the above as

$$
\operatorname{Tr}(U)=\sqrt{\frac{2 \pi}{i \hbar}} J_{0}(k / \hbar) \sum_{n=-\infty}^{\infty} \exp \left(2 \pi^{2} i n^{2} / \hbar\right)
$$

Strictly speaking this sum need not exist and may without further regularization be infinite. This is not due to any peculiarities of maps, and in fact this is the rule in quantum mechanics, the trace of the propagator could be infinite in real time. However this will not worry us further as the semiclassical trace will also diverge exactly, allowing us to make meaningful relative error estimates. 
Now we turn to a semiclassical evaluation of the quantum trace. We wish to then write the sum in terms of the period one or fixed points of the classical map. The Lagrangian generating the standard map on the cylinder is

$$
L\left(q_{n}, q_{n+1}, l_{n}\right)=\left(q_{n+1}-q_{n}+2 \pi l_{n}\right)^{2} / 2-k \cos \left(q_{n+1}\right)
$$

as $\partial L / \partial q_{n}=-p_{n}$ and $\partial L / \partial q_{n+1}=p_{n+1} . l_{n}$ is the winding number in the $q$ direction or $2 \pi l_{n}$ is subtracted by the modulo operation performed at time $n$ to keep the map on the cylinder. The fixed points are determined by the condition $\left(q_{n+1}=q_{n}, p_{n+1}=p_{n}\right)$, and are therefore simply given by $(q=0, p=2 \pi l)$ and $(q=\pi, p=2 \pi l)$, where $l$ runs over the integers. We note that the number of fixed points are infinite and that their positions do not depend on the classical chaos parameter $k$. The denominator in the Gutzwiller trace formula (or its versions for the case of quantum maps, they are essentially same) has the factor $\sqrt{\operatorname{Tr}(J)-2}$, where $J$ is the classical stability matrix associated with the periodic orbit. In the case of standard map fixed points this is either $\sqrt{k}$ or $\sqrt{-k}$ depending on whether $q=0$ or $q=\pi$.

The semiclassical approximation is of the form

$$
\operatorname{Tr}(U)_{s c}=\sum_{\text {fixed points }} \exp (i S / \hbar) / \sqrt{(\operatorname{Tr}(J)-2)}
$$

where $S$ is the classical action and since we are dealing with discrete time systems with the time interval being taken as unity this is identical to the Lagrangian written above. We get for the standard map on the cylinder the following semiclassical approximation for the trace (see Appendix):

$$
\operatorname{Tr}(U)_{s c}=\sqrt{\frac{4}{i k}} \cos \left(\frac{k}{\hbar}-\frac{\pi}{4}\right) \sum_{l=-\infty}^{\infty} \exp \left(2 \pi^{2} i l^{2} / \hbar\right)
$$

This then has to be compared with the Eq. (3). The asymptotic form of $J_{0}(x)$ for large arguments is [8]

$$
J_{0}(x) \sim \sqrt{\frac{2}{\pi x}}\left[\cos \left(x-\frac{\pi}{4}\right) P(x)-\sin \left(x-\frac{\pi}{4}\right) Q(x)\right]
$$

where

$$
P(x)=1-9 /\left(128 x^{2}\right)+\ldots \text { and } Q(x)=-1 /(8 x)+\ldots
$$

where the neglected terms are at most of order $x^{-3}$. We then derive that

$$
\frac{\operatorname{Tr}(U)}{\operatorname{Tr}(U)_{s c}}=P\left(\frac{k}{\hbar}\right)-\tan \left(\frac{k}{\hbar}-\frac{\pi}{4}\right) Q\left(\frac{k}{\hbar}\right) .
$$

Even this simple result is instructive. It indicates spectacular failures of the trace formula when the ratio $k / \hbar$ passes through $\pi / 4+(2 m+1) \pi / 2$, where $m$ is a positive integer. At these points the semiclassical trace becomes zero and the exact quantum trace is also small 
indicating that such a failure will not be serious for the evaluation of the spectra. But it does suggest that the ratio of the quantum to the semiclassical need not be bounded in the entire range of the parameters. This divergence of the ratio is thus not due to any coalescing stationary points or bifurcations in general; as we have already noted the fixed points are independent of the chaos parameter $k$. Second, it is clear that for a larger $k$ or chaos the semiclassical trace is going to be a better approximation. The authors in [2] have observed this, and we see that the simplest quantum trace provides a quantitative evidence of this. To compare the absolute error rather than the relative, we have to deal with the Jacobi theta function which occurs identically both in the quantum and in the semiclassical traces, and as this can depend critically on the rationality of $\pi / \hbar$ we do not pursue this here, except note that in unbounded phase spaces such as the plane and the cylinder the absolute value of the traces might actually diverge.

The case of the standard map has been extensively studied but not so the quantum mechanics of the sawtooth map, which is in many ways simpler because it is a piecewise linear map for which it is easy to extract classical information. Consider the map $\left(q_{n+1}=\right.$ $\left.q_{n}+T p_{n}, p_{n+1}=p_{n}+K\left(q_{n+1}-\pi\right)\right)$ defined on the cylinder $[0,2 \pi) \times(-\infty, \infty)$, which is completely chaotic for positive $K$. This can be considered as a simple harmonically kicked particle in one dimension. We may view the system as an otherwise free particle on a unit circle being subjected to a kick whose strength is proportional to the physical angle and which has a single discontinuity when the angle is zero. The trace of the quantum propagator may be written down just as in the case of the standard map $(T=1)$ with the result that

$$
\operatorname{Tr}(U)=\frac{1}{\sqrt{K}} \operatorname{Erf}\left(e^{-i \pi / 4} \sqrt{\frac{K}{2 \hbar}}\right) \sum_{n=-\infty}^{\infty} \exp \left(2 \pi^{2} i n^{2} / \hbar\right),
$$

where Erf is the error function.

The semiclassical trace is also found quite easily, because the fixed points are at $(q=$ $\pi, p=2 \pi l), l$ being any integer. The classical action is similar to that of the standard map with the potential term being replaced by a periodicised quadratic function. Thus we get

$$
\operatorname{Tr}(U)_{s c}=\frac{1}{\sqrt{K}} \sum_{n=-\infty}^{\infty} \exp \left(2 \pi^{2} i n^{2} / \hbar\right)
$$

and the ratio of the quantum to semiclassical simply becomes

$$
\frac{\operatorname{Tr}(U)}{\operatorname{Tr}(U)_{s c}}=\operatorname{Erf}\left(e^{-i \pi / 4} \sqrt{\frac{K}{2 \hbar}}\right)
$$

Inserting the asymptotic form for the error function we get the relative error to be

$$
\left|1-\frac{\operatorname{Tr}(U)}{\operatorname{Tr}(U)_{s c}}\right| \approx \sqrt{\frac{2 \hbar}{\pi K}} .
$$


The neglected terms are at most of order $(\hbar / K)^{3 / 2}$.

In this map also we see the seemingly general result that the larger the chaos (larger $K$ ) the semiclassical accuracy is better. The precise way in which this occurs for this map is like $K^{-1 / 2}$. The difference between this map and the standard map is that the semiclassical approximation seems more well behaved and the sudden decline in the accuracy in the case of the standard map, due to the mismatch between the zeros of the quantum and the semiclassical is absent here. However in the regions where the semiclassical (and quantum) traces are not very close to zero, the standard map would perform better as the errors are of order $\hbar / k$ while for the sawtooth map the errors are of order $(\hbar / k)^{1 / 2}$. We will see that surprisingly many of our observations will remain valid in the toral setting as well.

\section{The Toral Phase Space}

Two dimensional maps on the torus (periodic boundary conditions in both position and momentum) have been studied extensively, from the standard map to the cat map of Arnold. Their quantizations have also been investigated by many [3,7,9], as this is a legitimate model for a bound Hamiltonian system with two degrees of freedom, the minimum needed for chaos. Unlike the case of the cylindrical phase space where scattering may take place, sheer topology now prohibits this on the torus. The act of taking periodic boundary conditions on the momenta is reminiscent of a similar procedure in condensed matter physics which has also provided an interesting two dimensional map, namely the kicked Harper model. Quantum mechanics now is on a finite dimensional Hilbert space and the we take the torus to be a unit torus (the dissected square is of unit sides). An alternate view of toral quantum maps is as the case of "resonance" of the cylindrical map in which case the quantum mechanics can be exactly reduced to that of a torus. Position and momentum take discrete values which we take to be $n / N$, where $N$ is the dimension of the Hilbert space and $n=0 \ldots, N-1$. Here $N$ is required to be an even integer and is the inverse of the Planck constant. Therefore the classical limit is $N \rightarrow \infty$.

In this section we will first deal with the sawtooth map on the torus. We will write the map a little differently for convenience as $\left(q_{n+1}=\left(q_{n}+p_{n}\right) \bmod 1, \quad q_{n+1}=\left(p_{n}+\right.\right.$ $\left.K q_{n+1}\right) \bmod 1$.). The quantum map or propagator is given by

$$
U_{n m}=\frac{e^{-i \pi / 4}}{\sqrt{N}} \exp \left(i \pi K m^{2} / N\right) \exp \left(i \pi(n-m)^{2} / N\right) .
$$

This then is the quantum equivalent of the classical map restricted on a torus. It is a finite unitary matrix as all quantum maps on compact phase spaces are. Clearly it is one of the simplest quantum objects, having elementary functions as matrix elements. Yet we find that 
it is rich enough to reflect many essential features of the effect of classical chaos on quantum systems. For more properties of a very similar quantum map please refer [10].

The classical Lagrangian (or action) for this map is

$$
L\left(q_{n}, q_{n+1}, l_{n}, m_{n}\right)=\left(q_{n+1}-q_{n}+l_{n}\right)^{2} / 2+K\left(q_{n+1}(\bmod 1)\right)^{2} / 2-m_{n} q_{n+1}
$$

where the integers $l_{n}$ and $m_{n}$ are winding numbers in the $q$ and $p$ directions respectively. The fixed points are given by $(q=m / K, p=0)$ where $m=0,1,2, \ldots,[K]$. The fixed point at the origin is in fact on the line of discontinuity, and is quite like the bakers map and its quantization. In that case it was noted that such singular periodic orbits, "half hyperbolic ", could give rise to unusual semiclassical behaviour and that the quantum and semiclassical could diverge semiclassically as $\log \left(\hbar^{-1}\right)$. A bakers map was constructed to explicitly avoid this situation and a recovery of standard expectations was realized [6]. However if we take the above quantization as valid (since it follows canonical quantization closely [11]) it will turn out that the fixed point on the discontinuity is benign in the case of the sawtooth map, in that it does not lead to semiclassically divergent terms. This may suggest that the unusual nature of the bakers map originates either from the unusualness of the very quantization procedure adopted or is peculiar to the bakers map.

Let us look at this case more closely, and take $0<K<1$, so that there is a single fixed point at the origin which is also a point of discontinuity. A direct application of the Gutzwiller formula will be incorrect in this case, but the rectification is obvious. The stationary phase integral must not extend over the entire real line, but must be restricted to $[0, \infty)$. Thus the semiclassical approximation is then the approximation (quantum $\sim$ semiclassical)

$$
\frac{e^{-i \pi / 4}}{\sqrt{N}} \sum_{n=0}^{N-1} \exp \left(i \pi K n^{2} / N\right) \sim \frac{1}{2 \sqrt{K}} .
$$

An estimate due to Wilton which we give below bounds the difference as $N \rightarrow \infty$, but this is not a good estimate of the errors (for $0<K<1$ ), as these are of the order of the sums themselves. An application of the Euler-Maclaurin summation rule will give us the above approximation quite simply. But more important is the observation that it is legitimate to replace summations with integrals even in the presence of fixed points sitting on discontinuities, a rule that was flagrantly violated in the case of the bakers map.

Let the errors be $\epsilon(K, N)=\left|\operatorname{Tr}(U)-\operatorname{Tr}(U)_{s c}\right|$, that is the absolute value of the difference between the right hand and the left hand side of the Eq. (14). We now observe some remarkable number theoretic properties of these errors. First we note the surprising fact that if $N$ is a multiple of $4, \epsilon(1 / 2, N)=0$. In this case not only does the semiclassical and quantum converge in the classical limit, they are exactly the same (at the level of the trace), despite the presence of the fixed point on the discontinuity! We have noted before that the main 
feature which makes the sawtooth map generic, in the sense that the semiclassical is only an approximation, and in the sense of eigenfunctions scarring and spectral random matrix properties is that the incomplete Gauss sums that arise while compounding propagators do not reduce to simple forms [10], unlike in the case of the cat maps ( $K$ an integer) where we get complete Gauss sums and has been investigated by Hannay and Berry [9] and the extensive number theoretic properties were explored by Keating [12]. Thus it is a bit interesting that an incomplete Gauss sum can be done exactly and is the semiclassical approximation.

Consider the sum

$$
\sigma_{N}=\sum_{n=0}^{N-1} \exp \left(i \pi n^{2} / 2 N\right),
$$

which is incomplete because the period of $s_{n}=\exp \left(i \pi n^{2} / 2 N\right)$ is $2 N$ and the sum is only over the first $N$ terms. First we note that $s_{N / 2+m}=s_{N / 2-m} \exp (i \pi m)$, thus terms with odd $n$ do not matter as they cancel out of the sum exactly. Let

$$
\Sigma_{2 N}=\sum_{n=0}^{2 N-1} s_{n}
$$

which is a complete Gauss sum [9]. But also note that if $N$ is a multiple of 4 ,

$$
s_{n+N}=(-1)^{n} s_{n}
$$

This then implies that

$$
\sigma_{N}=\frac{1}{2} \Sigma_{2 N}=\frac{1}{2} \sqrt{2 N} e^{i \pi / 4}
$$

therefore

$$
\operatorname{Tr}(U)=e^{-i \pi / 4} \sigma_{N} / \sqrt{N}=1 / \sqrt{2}
$$

If $N$ is not divisible by 4 , then $\epsilon(1 / 2, N)$ is no longer zero and the semiclassical result is an approximation. Thus there are two distinct classes when $K=1 / 2$ as far as the behaviour of the error is concerned, This can be generalized and we observe that if $K=p / q$, is a rational number, with relatively prime integers $p$ and $q$, then there are as many classes as there are possible remainders of even $N$, on division by $q$. Within each class the error monotonically goes to zero in the classical limit, $N \rightarrow \infty$. We have observed numerically that for large $N$ to an excellent approximation

$$
\epsilon(K, N)=C(K) N^{-1 / 2}
$$

Fig. 1(a) shows the case $K=2 / 5$, when there are five classes. The constant $C(K)$ depends on both $K$ and the class, but we have observed that $C(K)=C(1-K)$. If $K$ is irrational, the number of classes is infinite, there is no monotonic decrease of error, but the general trend is to decrease, as is evident from Fig. 1(b), where we have taken $K$ to be the 


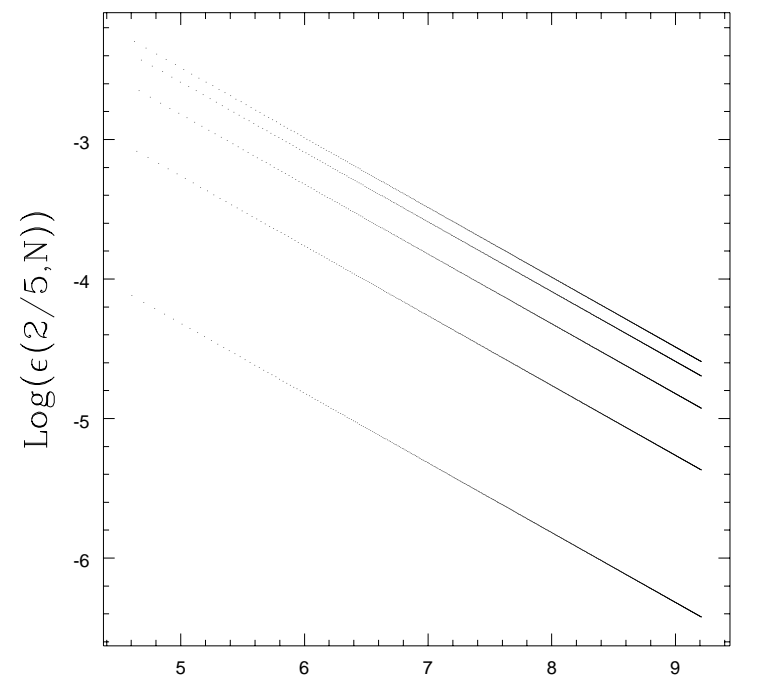

a) $\log (\mathrm{N})$

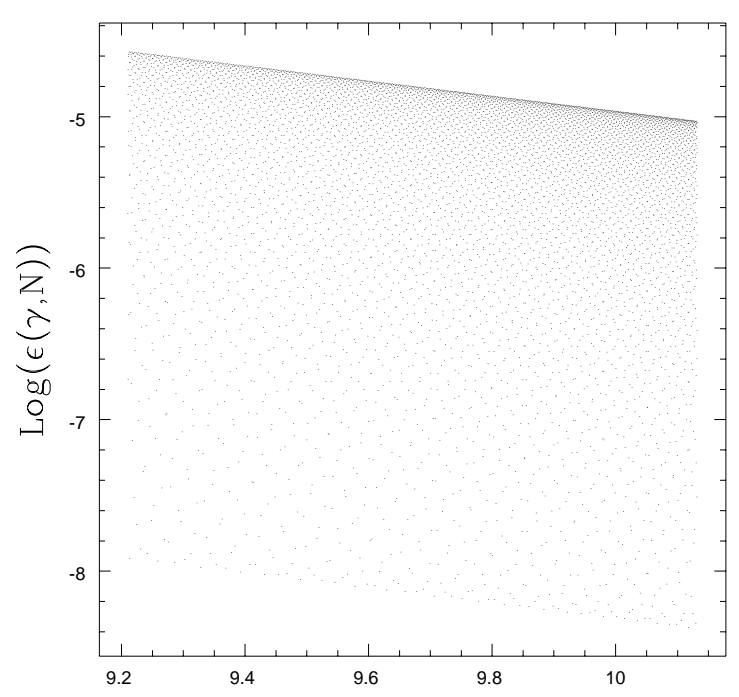

b) $\log (\mathrm{N})$

Figure 1: The error as a function of inverse Planck's constant $N$, for the sawtooth map with (a, left) $K=2 / 5$; (b, right) $K=\gamma$.

most irrational number $\gamma=(\sqrt{5}-1) / 2$, the golden mean. The number theoretical properties of the error is of course a reflection of the sum itself, and a fuller study is thus desirable.

We however shall now turn to the case when $K$ is larger than one, and there are a number of fixed points. If $K$ is much larger than unity we need not correct for the corner fixed point as the errors incurred will be of the order $K^{-1 / 2}$, which is anyway the order of the total error according to Hardy, Littlewood and Wilton, as quoted in [13]. The semiclassical approximation is now described by

$$
\frac{e^{-i \pi / 4}}{\sqrt{N}} \sum_{n=0}^{N-1} \exp \left(i \pi K n^{2} / N\right) \sim \frac{1}{\sqrt{K}} \sum_{n=0}^{[K]} \exp \left(-i \pi N n^{2} / K\right) .
$$

Here $[K]$ is the integer value of $K$. Note that this approximation clearly demonstrates the conjugacy between the Planck constant $(1 / N)$ and the chaos parameter $K$. It is a good approximation if $K$ is fixed and $N$ tends to infinity, namely the classical limit, but also in the opposite limit of extreme "chaos", and small quantum system. For example when $K$ tends to infinity for $N=2$, a two level system. Undoubtedly, this approach to two level systems is to say the least inadvisable, as the quantum problem is much easier to solve. Nevertheless it throws up some interesting questions, because what would one mean by extreme chaos in a two level system? Quantum chaos for legitimacy in using the epithet chaos has always relied on the classical limit, and in the extreme quantum limit the concept becomes nebulous.

The above approximation is derived from the Gutzwiller formula, using the $[K]+1$ fixed 
point actions and the stability matrix. The fixed point at the origin is included wrongly in the formula and we have discussed it separately in the case $0<K<1$ above. Now we use an estimate of Wilton, quoted in [13], to bound the error in this approximation. This estimate is the inequality

$$
\left|\operatorname{Tr}(U)-\operatorname{Tr}(U)_{s c}\right|<\frac{a}{\sqrt{N}}+\frac{b}{\sqrt{K}}
$$

where $a$ and $b$ are constants of order one whose values are slightly different from the quoted ones, as the sawtooth sum is from 0 to $N-1$, rather than 1 to $N$, but this is a trivial complication. The essential conclusion is that the error is bounded in $N$, and corroborates our conclusion that there is no $\log (N)$ problems in the sawtooth map despite the presence of the fixed point on the singularity. Also we once again notice that a larger $K$ (larger chaos) implies a better semiclassical accuracy. In Fig. 2 we show the results of a numerical computation of the error, which shows that the error goes roughly as $K^{-1 / 2}$, with the slope depending weakly on $N$ and $K$ and rather strongly on the fractional part of $K$. Notice that at integer $K$ (cat maps) the approximation is exact. We thus note that like its relative on the cylinder the torus map error is also of the order of $K^{-1 / 2}$.

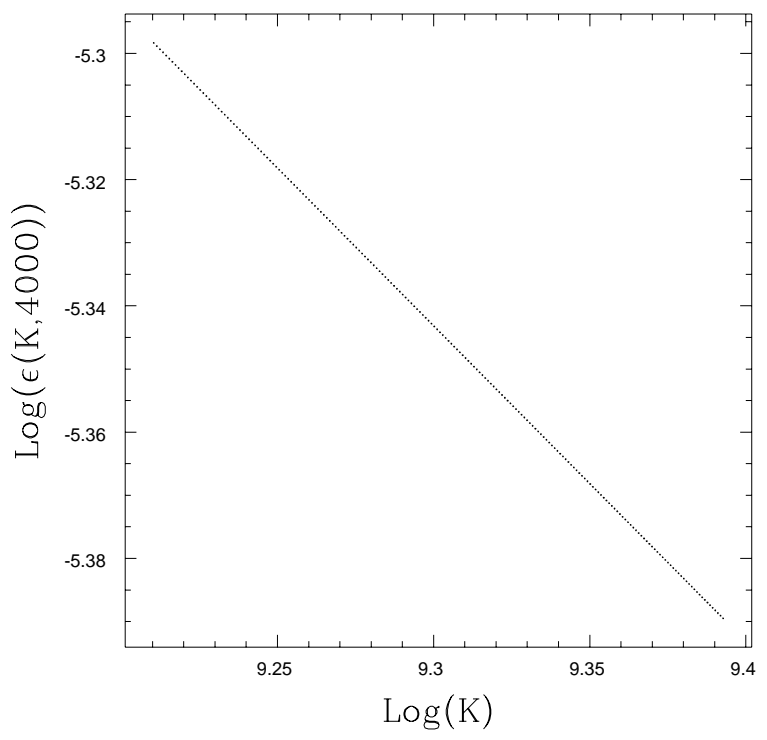

Figure 2: The error as a function of the chaos parameter $K$, for the sawtooth map with $N=4000$.

We had earlier studied the sawtooth map on the torus with slightly different boundary conditions [10], and came to essentially similar conclusions, however in that case there was no fixed point on the discontinuity. When the fixed points moved towards the singularity, 
at the exact point of contact, the map turned into the continuous cat map when the trace formula becomes exact. We now turn to a smooth map on the two torus, the standard map. Much studied in other contexts, it is largely unstudied from the view of trace formulas, although it should form a natural object of study, as it represents generic mixed systems, and one recognizes the difficulties of such systems apropos trace formulas.

We will consider the map once again on the unit torus. The map is now $\left(q_{n+1}=\left(q_{n}+\right.\right.$ $\left.\left.p_{n}\right) \bmod 1, p_{n+1}=\left(p_{n}+k \sin \left(2 \pi q_{n+1}\right)\right) \bmod 1\right)$. The Lagrangian (action) is

$$
L\left(q_{n}, q_{n+1}, l_{n}, m_{n}\right)=\left(q_{n+1}-q_{n}+l_{n}\right)^{2} / 2-k \cos \left(2 \pi q_{n+1}\right) / 2 \pi-m_{n} q_{n+1} .
$$

The fixed points are easy to find and are at $\left(q_{m}=\frac{1}{2 \pi} \arcsin (m / k), p_{m}=0\right)$, where $\mathrm{m}$ is an integer such that $|m|<k$. Once again the location and number of the fixed points are very much dependent on the the chaos parameter $k$ unlike the standard map on the cylinder. Note that there are two fixed points associated with each $m$.

The quantum propagator is again given by ( $N$ even throughout)

$$
U_{n m}=\frac{e^{-i \pi / 4}}{\sqrt{N}} \exp (-i k N \cos (2 \pi n / N)) \exp \left(i \pi(n-m)^{2} / N\right) .
$$

and the trace is the finite sum

$$
\operatorname{Tr}(U)=\frac{e^{-i \pi / 4}}{\sqrt{N}} \sum_{n=0}^{N-1} \exp (-i k N \cos (2 \pi n / N)) .
$$

The semiclassical approximation must approximate this sum using the classical fixed points, their actions and their stabilities. First note that the quantum trace apart from the factor $e^{-i \pi / 4}$ is real. The sawtooth map traces were Gaussian sums, and partial sums of these give rise to the "curlicues", that have been studied in detail earlier and find applications from optics to quantum mechanics [13]. The partial sums associated with the quantum trace of the standard map ought to be interesting mathematical objects in their own right.

Here we present a preview of the intriguing class of periodic "curves" that this gives rise to. Consider the sum

$$
\sigma_{N}(k, l)=\sum_{n=0}^{l} \exp (-i k N \cos (2 \pi n / N)) .
$$

The quantum standard map trace is essentially $\sigma_{N}(k, N)$. We plot in Fig. 3 the real, $x(l)$, and imaginary, $y(l)$, parts of $\sigma_{N}(k, l)$ as an argand diagram. Strictly there are only as many points as terms in the partial sum, and as such do not define a curve, but the connections showing the motion of the points in "time" $l$ is suggestive. Only the sum up to $l=N / 2$ is relevant as beyond this a reflection and periodic translation is the whole curve. Hence unlike curlicues, these are periodic curves. A plethora of cornu spirals interweave to conjure the extravagantly ornate features. Several "eyes" of cornu like spirals are connected in a 
remarkable manner. An essential feature of these curves is their extreme susceptibility to small changes in $k$, especially at large $N$ values. We cannot rule out the possibility of number theoretic features here as well, and indeed we observe marked difference between the cases when $k$ is an integer and when it is not.

The periodic nature of the curves are easily understood, as $y(l+N / 2)=-y(l)$ and $x(l+N / 2)=x(l)+A$, where $A=\sum_{n=0, N / 2-1} \cos (-i k N \cos (2 \pi n / N))$. Hence the period of the curves are $2 A$. As $N$ is increased for fixed $k$ the curves tend to smoothen out more but with the cornu spiral structures intact. Also note that the quadratic approximation of the cosine term in the exponential are the culicues studied earlier [13].
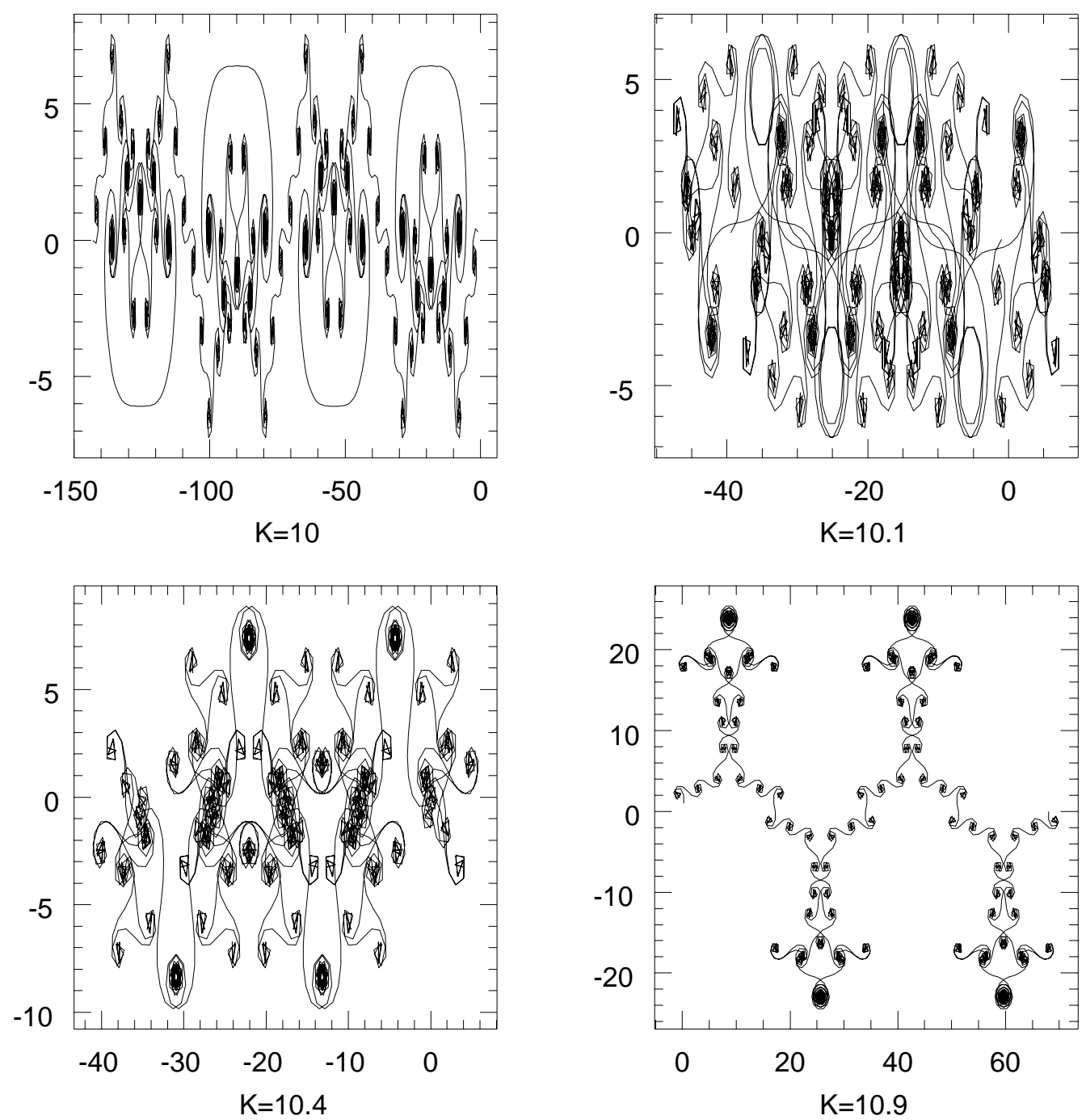

Figure 3: A panel of the partial sums $\sigma_{1000}(K, l)($ Eq. 23) for various $K$. 
A detailed study of these curves is however not pursued in this paper, and we turn once more to the central theme, namely the accuracy of semiclassical approximations. Define

$$
\theta_{N}(l)=N\left(\sqrt{k^{2}-l^{2}}+l \arcsin (l / k)\right)
$$

Then a careful application of the trace formula yields the semiclassical approximation

$$
\operatorname{Tr}(U)_{s c}=\frac{e^{-i \pi / 4}}{\sqrt{2 \pi}}\left[\frac{2 \sin (N k+\pi / 4)}{\sqrt{k}}+\sum_{l=1}^{[k]} \frac{4 \sin \left(\theta_{N}(l)+\pi / 4\right)}{\left(k^{2}-l^{2}\right)^{1 / 4}}\right],
$$

which is real except for the factor $e^{-i \pi / 4}$, just as the exact quantum trace in Eq. (22). Here the fixed point at the origin has been treated separately as corresponding to each $|m|$ there are four fixed points except when $m=0$, which gives rise to two fixed points. The range of the inverse sine function is $[0, \pi / 2]$. Note that this approximation can be derived independent of the trace formula, by first converting the sum into an infinite sum of integrals via the Poisson summation formula, and of these integrals retaining only those which contribute to the stationary phase, and evaluating these integrals in the quadratic approximation. Indeed the trace formula in the context of maps on the torus is derivable by this procedure and is hence considerably simpler than the derivation for general Hamiltonian flows (see Appendix).

First we note a drawback of the semiclassical approximation. Near parameter values where bifurcations are going to create new periodic points the semiclassical approximation becomes poor. Uniform approximations may be made to smooth over these parameter values. For instance in the case of the standard map, whenever $k$ passes an integer value it creates four new fixed points via tangent bifurcations, and the approximation to the trace becomes poor. This is illustrated in Fig. 4(a). However notice that the approximation is indeed quite good even just away from these values. We will below retain the approximation made above and keep away from points of bifurcations to study various aspects of the error.

In Fig. 4(b) we show the absolute value of the error $\epsilon=\left|\operatorname{Tr}(U)-\operatorname{Tr}(U)_{s c}\right|$ for a fixed value of $k=100.5$, and a range of $N$. It is clear that the errors involved are not monotonically decreasing with $N$, but that there are fluctuations in the mean. The mean however very nearly follows $N^{-1}$ behaviour. This should be contrasted with the more cleaner but slower $N^{-1 / 2}$ behaviour of the sawtooth map. In general the errors incurred for the smooth standard map is smaller than those of the sawtooth map. We see that this is a property that is carried over from the maps on the cylinder. It is unclear whether the standard map fairs better due to its smoothness or due to some other property. This said, we note that the trace formula approximation behaves better with increasing chaos in the case of the sawtooth map than the standard map.

At a fixed value of $N$ the behaviour of the trace for increasing $k$ is quite complex. No simple rule like, higher chaos implies better semiclassical accuracy emerges. First we note 

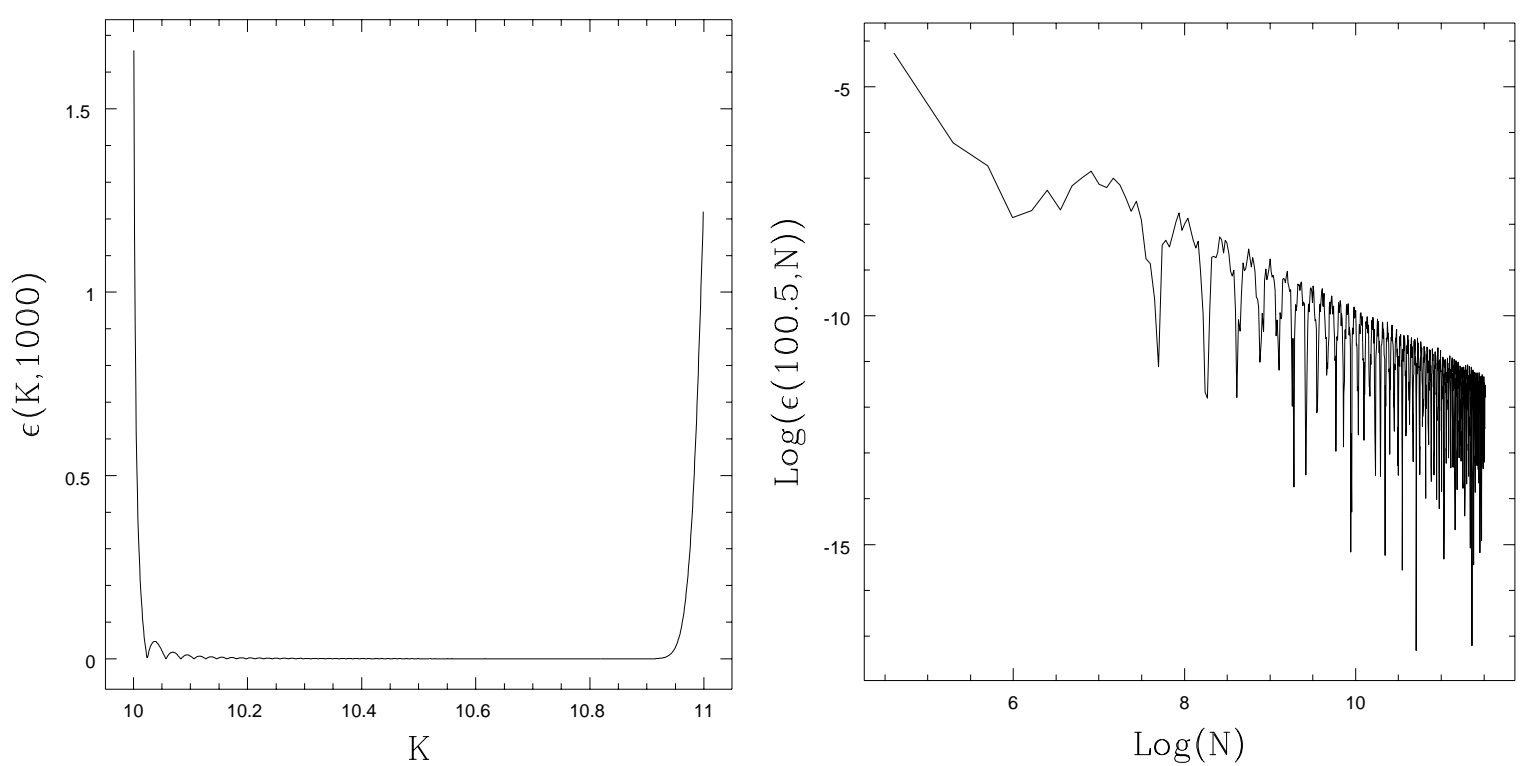

Figure 4: The error for the standard map on the torus as a function of (a, left) $k$, with $N=1000$; (b, right) inverse Planck's constant $N$, with $k=100.5$

that the ratios or the relative error fair quite poorly, with large abrupt deviations. Fig. $5(\mathrm{a})$ shows the relative errors $|\epsilon| /\left|\operatorname{Tr}(U)_{s c}\right|$, but the range in the $y$ axis has been lessened to highlight the predominant features. The error could become as high as 40 in the range of $k$ shown. This is however easy to understand in the context of the Eq. (7) for the map on the cylinder, as due to the mismatch of the zeros of the semiclassical and the quantum traces. We expect the absolute value of the errors to fair better. Indeed when $N$ is small, one observes an increase of accuracy with $k$, and this is a power law for small $N$. Thus we show in Fig. 5(b) the logarithm of the errors with the logarithm of $k$, and we observe the rule that $\epsilon=C k^{-\gamma}$, with $\gamma \sim .25$, for the extreme quantum case $N=2$. Here the $k$ go in steps of unity with their fractional part being uniformly .5 .

However as $N$ is increased we observe that this is no longer true, and that the errors tend to oscillate with $k$ in a fairly complex manner. The overall tendency seems to be in fact a deterioration of the accuracy with $k$. Fig. 8 shows the errors as a function of $k$ in a large range. The interesting aspect is that the oscillation period seems to increase geometrically. Therefore there is a possibility that the errors scale with $k$, but in a complex manner. However increasing $k$ even higher seems to render the errors an almost monotonic growth, and we cannot comment on the many details until further analytic estimates are made and computer generated errors are under control.

An important difference between the semiclassics of the sawtooth map and the standard 

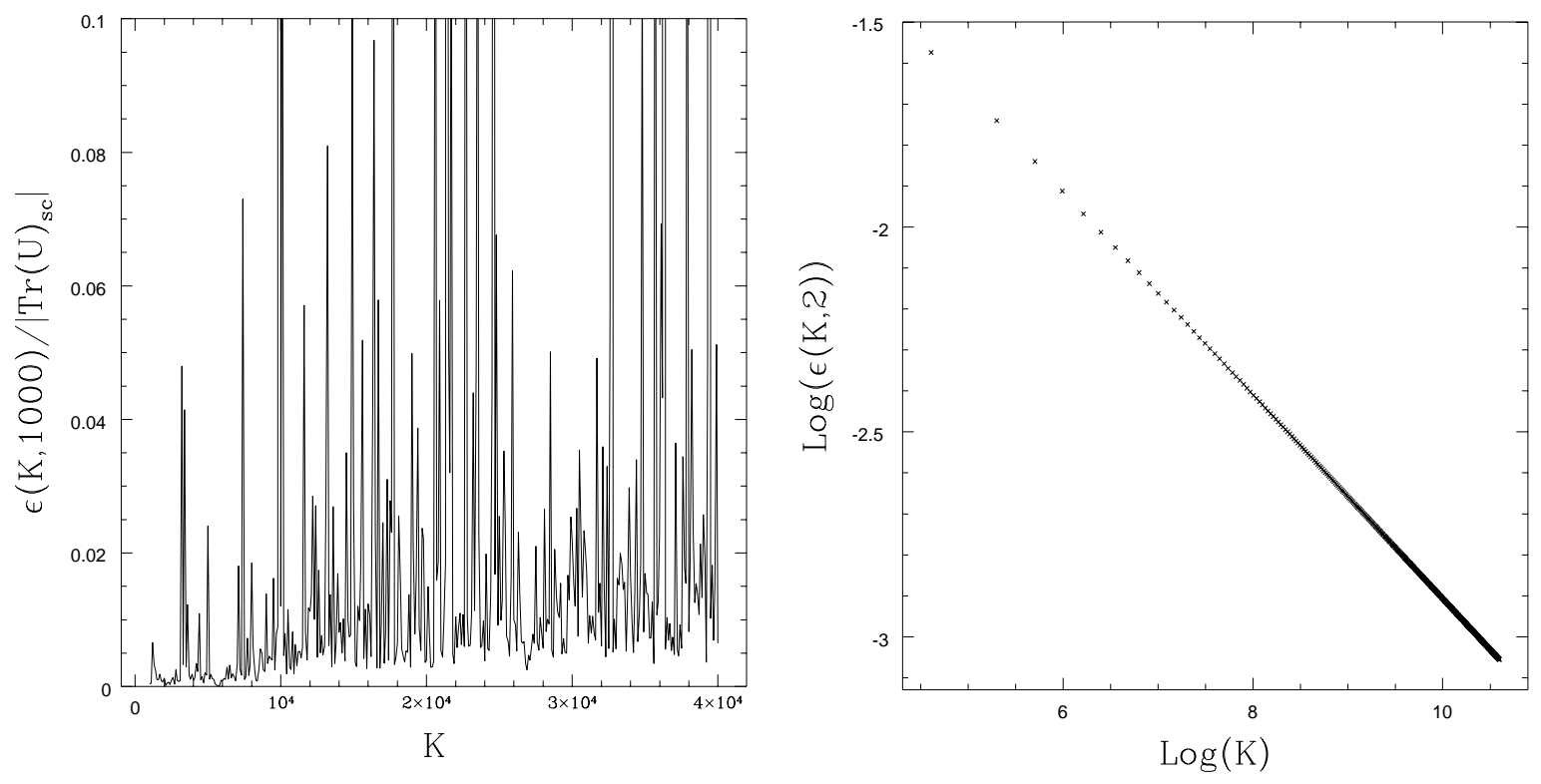

Figure 5: (a, left) The relative error for the standard map, as a function of the chaos parameter $k, N=1000$; (b, right) the absolute error as a function of $k$ in the extreme quantum limit, $N=2$.

map is that because of the piecewise linear nature of the sawtooth map there was no need to truncate Taylor expansions about the periodic orbits, while in the case of the standard map this truncation is done up to second order. Higher order corrections can be worked out and have formed the subject of recent works $[15,16]$, it should be interesting to compare the errors when such higher order terms are included.

\section{Summary}

Using maps as models of quantum chaos we have studied the simplest traces from the point of view of semiclassical approximations, hoping that this will reflect some more general aspects of errors involved in the Gutzwiller's periodic orbit sum or trace formula. We found that indeed the maps on the cylinder had controllable relative errors. We found that the smooth standard map faired better than the sawtooth map in the order of magnitude of the errors. We also noted that the mismatch of zeros between the semiclassical and the quantum can lead to deterioration of relative errors. In the case of toral maps we displayed a rather remarkable fact that even in the presence of fixed points on discontinuities the trace formula may be exact at the level of the time one trace. Otherwise we showed that more generally there is no $\log (\hbar)$ divergences in the sawtooth map, unlike the case of the quantum bakers map. We also noted that the error may actually decrease with increasing chaos, a rather 


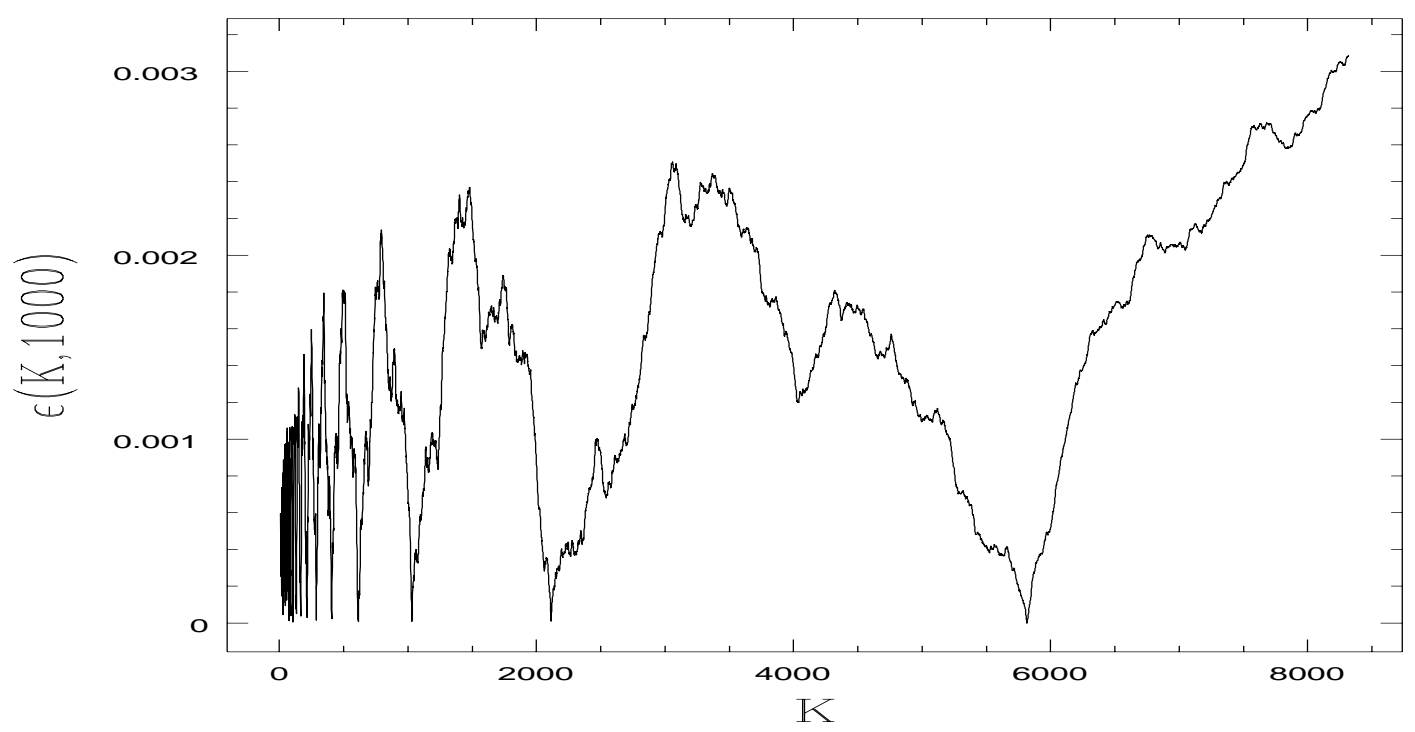

Figure 6: The error as function of $k$ in a wide range of $k$, for the standard map with $N=1000$.

counter intuitive fact, which has quantitative evidence in the case of the sawtooth map. However we caution that this cannot be a general principle as a study of the standard map has shown a much more complex behaviour of the error. It is indeed a matter of importance to study and contrast the two maps, and understand why the errors behave qualitatively differently.

We have restricted our study to the time one trace and indeed it is natural and necessary that we study the higher traces. For instance to study the errors involved in the semiclassical evaluation of $\operatorname{Tr}\left(U^{2}\right)$ leads us into the problem of enumerating period two orbits, which can be fairly easily computed in the cases studied, but which are much larger in number than the fixed points. Preliminary results in this direction indicate that for the sawtooth map the error behaviour of the time two trace is similar to that of the trace, although the errors are larger. We have also displayed some remarkable curves akin to curlicues that arise out of a study of the trace of the standard map on the torus. 


\section{References}

[1] M.C. Gutzwiller, "Chaos in Classical and Quantum Mechanics", Springer (New York, 1990).

[2] M.A. Sepulveda, S.Tomsovic and E.J. Heller, Phys. Rev. Lett. 69 (1992), 402.

[3] N.L. Balazs, and A. Voros, Ann. Phys., (N.Y.)190 (1989), 1.

[4] P.W.O' Connor, S. Tomsovic, and E.J. Heller, Physica 55D (1992), 340; A.M.O. De Almeida, and M. Saraceno, Ann. Phys., (N.Y.) 210 (1991), 1; M. Saraceno, Ann. Phys., (N.Y.) 199 (1990), 37.

[5] M. Saraceno, and A. Voros, Physica 79D (1994), 206.

[6] A. Lakshminarayan, Ann. Phys., (N.Y.) 239 (1995), 272.

[7] F. M. Izrailev, Phys. Rep. 196 (1990), 299.

[8] G. N. Watson, "A Treatise on the Theory of Bessel Functions", Cambridge University Press (London, 1966).

[9] J.H. Hannay, and M.V. Berry, Physica 1D (1980), 267.

[10] A. Lakshminarayan, Phys. Lett. A192 (1994), 345; A. Lakshminarayan and N. L. Balazs, Chaos, Solitons, Fractals (Osaka), 5 (1995), 1169.

[11] J. Ford, G. Mantica, and G.H. Ristow, Physica 50D (1991), 493.

[12] J. P. Keating, Nonlinearity 4 (1991), 335.

[13] M. V. Berry, and J. Goldberg, Nonlinearity 1 (1988), 1.

[14] J. M. Greene, J. Math. Phys. 20 (1979), 1183.

[15] P. Gaspard, and D. Alonso, Phys. Rev. A47 (1993), R3468.

[16] G. Junker and H. Leschke, Physica D56 (1992), 135.

[17] M. Tabor, Physica D6 (1983), 195. 


\section{Appendix}

Consider the following map defined on the unit torus:

$$
\begin{aligned}
q_{j+1} & =q_{j}+p_{j}-l_{j} \\
p_{j+1} & =p_{j}-V^{\prime}\left(q_{j+1}\right)-m_{j},
\end{aligned}
$$

where $V(q)$ is a periodic "kicking" potential with unit periodicity, the prime indicates derivative, and the integers $m_{j}$ and $l_{j}$ are such that the map is restricted to the unit torus (winding numbers). They are the "modulo one" operations. As is well known the above map can be derived from a time dependent Hamiltonian system. Also there exists a generating function for the map on the torus given by

$$
S\left(q_{j}, q_{j+1} ; l_{j}, m_{j}\right)=\frac{1}{2}\left(q_{j+1}-q_{j}+l_{j}\right)^{2}-V\left(q_{j+1}\right)-m_{j} q_{j+1}
$$

from which the map can be derived as $p_{j}=-\partial S / \partial q_{j}$ and $p_{j+1}=\partial S / \partial q_{j+1}$.

The quantum map corresponding to the Eqs.(26) is given by the finite dimensional unitary matrix

$$
\left\langle n|U| n^{\prime}\right\rangle=\frac{e^{-i \pi / 4}}{\sqrt{N}} \exp \left(\frac{i \pi}{N}\left(n-n^{\prime}\right)^{2}-2 \pi i N V\left(\frac{n}{N}\right)\right) .
$$

This takes on the function of the propagator as it connects states after consecutive kicks. Here the representation is in the position basis and $n$ and $n^{\prime}$ may take the integer values from 0 to $N-1$. $N$ is itself the dimensionality of the Hilbert space and is also the inverse Planck constant, and is restricted to be even integers for preservation of toral boundary conditions. Semiclassics means the study of the unitary operator as $N \rightarrow \infty$.

The object of primary interest below is the trace of the powers of the propagator from which the spectrum may be derived by a Fourier transform. This can be written as

$$
\operatorname{Tr}\left(U^{T}\right)=\frac{e^{-i \pi T / 4}}{N^{T / 2}} \sum_{n_{i}=0}^{N-1} \exp \left(\frac{2 \pi i}{N} \sum_{j=1}^{T}\left(n_{j}^{2}-n_{j} n_{j+1}-N^{2} V\left(\frac{n_{j}}{N}\right)\right)\right),
$$

with

$$
n_{T+1}=n_{1}
$$

It is implied in the above that the outer sum is over the $T$ variables $n_{i}, i=1,2, \ldots, T$. Using the Poisson summation formula we can rewrite the above as

$$
\begin{array}{r}
\operatorname{Tr}\left(U^{T}\right)=e^{-i \pi T / 4} N^{T / 2} \sum_{k_{i}=-\infty}^{\infty} \int_{-\epsilon}^{1-\epsilon} d x_{1} \ldots d x_{T} \\
\exp \left(2 \pi i N \sum_{j=1}^{T}\left(k_{j} x_{j}+x_{j}^{2}-x_{j} x_{j+1}-V\left(x_{j}\right)\right)\right),
\end{array}
$$


with

$$
x_{T+1}=x_{1} \text {, and } k_{T+1}=k_{1} .
$$

The stationary phase approximation, assuming that $N$ is large, gives the following $T$ conditions

$$
x_{j+1}=2 x_{j}-x_{j-1}-V^{\prime}\left(x_{j}\right)+k_{j} ; \quad j=1, \ldots, T, \quad x_{0}=x_{T}, x_{1}=x_{T+1},
$$

with the further condition that

$$
0 \leq x_{j}<1
$$

These are precisely the equations that determine the period $T$ orbits of the map. The integers $k_{j}$ are related to the integers $l_{j}$ and $m_{j}$ of Eqs. (26) by the relation $k_{j}=l_{j-1}-l_{j}-m_{j-1}$. With the assumption that they uniquely determine a periodic orbit of period an integer fraction of $T$, we restrict the infinite sum to only those that relate to such a periodic orbit labeled below by $\beta$. This is the first approximation and the second one is that we will extend the ranges of integration to the whole real line. These approximations together are exact in the case of the cat maps $[9,11,12]$, and the third related approximation is that we Taylor expand the potential about the periodic orbits retaining up to the quadratic terms. This approximation is unnecessary for the piecewise linear sawtooth maps [10], but is necessary in general.

Therefore we define new variables $y_{j}$ such that $x_{j}=q_{j}+y_{j}$ and $q_{j}$ is the central periodic orbit.

$$
\begin{array}{r}
\operatorname{Tr}\left(U^{T}\right) \sim e^{-i \pi T / 4} N^{T / 2} \sum_{\beta} \exp \left(2 \pi i N \sum_{j=1}^{T}\left(-q_{j} q_{j+1}+q_{j}^{2}+q_{j} k_{j}-V\left(q_{j}\right)\right)\right) \times \\
\int_{-\infty}^{\infty} d y_{1} \ldots d y_{T} \exp \left(2 \pi i N \sum_{j=1}^{T}\left(y_{j}^{2}-y_{j} y_{j-1}-y_{j}^{2} V^{\prime \prime}\left(q_{j}\right) / 2\right)\right)
\end{array}
$$

The sum

$$
\sum_{j=1}^{T}\left(-q_{j} q_{j+1}+q_{j}^{2}+q_{j} k_{j}-V\left(q_{j}\right)\right)
$$

can be identified with the action of the periodic orbit, as from Eq.(27) one gets

$$
S_{\beta}=\sum_{j=1}^{T}\left(q_{j}^{2}-q_{j} q_{j+1}-V\left(q_{j}\right)+q_{j} k_{j}+l_{j}^{2} / 2\right),
$$

which is essentially the sum in Eq. (33). We can neglect the term $l_{j}^{2} / 2$, from the Lagrangian; this is legitimate as the action occurs as $e^{2 \pi i N S}$, and since $N$ is an even integer these terms do not matter. Note that the trace formula at $T=1$ is easily obtained from the above and has been used in the main body of this paper. 
The integral is evaluated by standard methods.

$$
\int_{-\infty}^{\infty} d^{T} y \exp \left(i \pi N y^{t} A_{\beta} y\right)=\frac{e^{i \pi T / 4}}{N^{T / 2}} \frac{e^{-i \pi \nu_{\beta} / 2}}{\sqrt{\left|\operatorname{Det}\left(A_{\beta}\right)\right|}}
$$

Here $y$ denotes the $T$ component vector $\left\{y_{j}\right\}$ and $y^{t}$ is its transpose. The real symmetric matrix $A_{\beta}$ is given by

$$
A_{\beta}=\left(\begin{array}{cccccc}
2-V^{\prime \prime}\left(q_{1}\right) & -1 & 0 & \ldots & \ldots & -1 \\
-1 & 2-V^{\prime \prime}\left(q_{2}\right) & -1 & 0 & \ldots & 0 \\
: & : & : & : & : & : \\
: & : & : & : & : & : \\
: & : & : & : & : & : \\
-1 & 0 & \ldots & \ldots & -1 & 2-V^{\prime \prime}\left(q_{T}\right)
\end{array}\right) .
$$

$\nu_{\beta}$ is a "Maslov like" index, and is the number of negative eigenvalues of the matrix $A_{\beta}$.

The nontrivial problem of evaluating the determinant is already solved and the results are well known [14]. The determinant can be related to the stability of the periodic orbit. If the residue of the periodic orbit, which is simply related to the trace of the stability matrix, is $R_{\beta}$, then $R_{\beta}=-\frac{1}{4} \operatorname{Det}\left(A_{\beta}\right)$. or if we denote the stability matrix eigenvalues by $\lambda_{+}^{\beta}$ and $\lambda_{-}^{\beta}, \operatorname{Det}\left(A_{\beta}\right)=\lambda_{+}^{\beta}+\lambda_{-}^{\beta}-2$.

Thus finally we can write the asymptotic periodic orbit sum, or the trace formula, for the toral maps as

$$
\operatorname{Tr}\left(U^{T}\right) \sim \sum_{\beta} \frac{\exp \left(2 \pi i N S_{\beta}-i \pi \nu_{\beta} / 2\right)}{\sqrt{\left|\lambda_{+}^{\beta T}+\lambda_{-}^{\beta T}-2\right|}} .
$$

The sum is over periodic orbits whose periods are integer fractions of $T$ are labeled by $\beta$. This is of the canonical Gutzwiller-Tabor form having in the exponent the action of the period $T$ orbits, and the prefactor explicitly depending on the stability of these orbits. The piecewise linear maps such as the baker's map, or the sawtooth map are such that the Maslov like phases are zero and the only type of periodic orbits are those of the direct hyperbolic kind. Now however we can study the vast class of mixed systems, such as the well known standard map. It is then of interest to ask questions such as how long the semiclassical approximations are valid in such systems which one knows are generic. The quantum mechanics on the torus is exact and thus we expect that the derivation above will facilitate the investigation of the periodic orbit sum for mixed systems. In this note we have used the above formula only in the special case of $T=1$. 